\title{
Calibration and Comparison of Chlorine Decay Models for a Test Water Distribution System
}

\author{
Stefan H. Maier*, Roger S. Powell* and Clive A. Woodward $\dagger$,
}

*Water Operational Research Centre, Department of Manufacturing and Engineering Systems, Brunel University, Uxbridge, UB8 3PH, U.K.; †Thames Water Utilities Ltd., Group Research \& Technology, Spencer House, Manor Farm Road, Reading, RG2 0JN, U.K.

All correspondence to be sent to: Dr Roger Powell, Water Operational Research Centre, Department of Manufacturing and Engineering Systems, Brunel University, Uxbridge, UB8 3PH, U.K. Tel. 01895/203296, Fax. 01895/812556, email roger.powell@brunel.ac.uk.

Short Title: Comparison of Chlorine Decay Models

Keywords: water distribution system, water quality, chlorine decay coefficients, network calibration, parameter estimation, EPANET

\begin{abstract}
This paper investigates the kinetics of monochloramine as disinfectant in a $1.3 \mathrm{~km}$ water pipe. A novel procedure for the correction of chlorine meter errors is introduced and applied. Parameter estimation using nonlinear optimisation procedures is used to identify decay coefficients for monochloramine models with a single coefficient or two coefficients as used in EPANET. Important difficulties in fitting these parameters which come about because of the model structure are highlighted. Finally, results of decay coefficients are presented and investigated for flow, inlet chlorine concentration and temperature dependence.
\end{abstract}

\section{Introduction}

Free chlorine and monochloramine are widely used as disinfectants in water distribution systems. Knowledge of the dynamic behaviour of this disinfectant is important when designing new systems and when determining the effects of operational changes. 
The aim of this paper is to investigate and compare two commonly used chlorine decay models in detail, i.e. a single decay coefficient model and the combined bulk and wall demand model as used in EPANET (?), using data obtained from physical measurements. The models are adapted for the use of monochloramine as disinfectant. In addition, data from bottled samples are presented and compared with results obtained from the chlorine transducers.

\section{Review of Chlorine Kinetics}

Most work on disinfection kinetics is concerned with free chlorine $\left(\mathrm{Cl}_{2}\right.$ or $\left.\mathrm{HOCl}\right)$. It is frequently assumed that free chlorine decays according to first order reaction kinetics (?; ?):

$$
c\left(x_{2}, t_{2}\right)=c\left(x_{1}, t_{1}\right) \cdot \mathrm{e}^{-K\left(t_{2}-t_{1}\right)}
$$

with

$$
x_{2}-x_{1}=v \cdot\left(t_{2}-t_{1}\right),
$$

where $t_{1}, t_{2}$ represent time, $x_{1}, x_{2}$ represent distance along the pipe and $v$ is the constant velocity of water in the pipe. Therefore, the decay of free chlorine is characterised by just one (constant) decay coefficient $K$. The travel time between points $x_{1}$ and $x_{2}$ is $\tau=\frac{x}{v}=t_{2}-t_{1}$.

A variation of the decay coefficient $K$ may be required, since in recent years more evidence has been published that shows that simple first order kinetics do not fully match the behaviour of free chlorine in a distribution system. Lungwitz et al. (?) investigated booster (impulse) chlorination and found that they had to 
postulate several decay coefficients depending on the chlorine gradient. Schneider et al. (?) concluded that the decay coefficient (and possibly other parameters) are flow dependent. Woodward et al. (?) found experimentally that the monochloramine decay coefficient changes with flow rate. Heraud et al. (?) used different decay coefficients depending on pipe materials and diameter to fit their model to the data.

Simulation packages reflect these findings to some extent. In Piccolo (?) it is possible to use a chlorine decay coefficient, $K_{H_{2} 0}$, which depends on total organic carbon and temperature or to use diameter dependent decay coefficients within the distribution system. EPANET (?; ?) includes a wall demand and a flow dependent mass transfer coefficient. Thus, it replaces the constant decay coefficient $K$ with

$$
K=k_{b}+\frac{k_{f} k_{w}}{R_{H}\left(k_{f}+k_{w}\right)}
$$

where $k_{f}$ is a flow dependent mass transfer coefficient, $k_{b}, k_{w}$ are parameters representing bulk and wall demand respectively and $R_{H}$ is the hydraulic radius. The mass-transfer coefficient $k_{f}$ is calculated as given in Rossman (?) or Edwards et al. (?).

In the present study, monochloramine is investigated since it is used in the London distribution system and it also finds application in other water networks world-wide, e.g. (?). Monochloramine $\left(\mathrm{NH}_{2} \mathrm{Cl}\right)$ is generally believed to behave in the same fashion as free chlorine, except that it reacts more slowly (?); also it was suggested that monochloramine is more efficient against biofilms than free chlorine (?; ?). 
Total chlorine, which includes free chlorine, monochloramine and organic chloramines, is measured using online meters. Organic chloramines are relatively stable and have a very low bacterial action (?) and thus are considered as a constant fraction of total chlorine in this work.

Thus, first order kinetics as in Fig. ?? are obtained, which can be described as

$$
c\left(x_{2}, t_{2}\right)=\left(c\left(x_{1}, t_{1}\right)-c_{\infty}\right) \cdot \mathrm{e}^{-K\left(t_{2}-t_{1}\right)}+c_{\infty} .
$$

where $c(x, t)$ is the total chlorine concentration at position $x$ and time $t$.

Monochloramine is the reactive fraction of total chlorine, or $\left\{c(x, t)-c_{\infty}\right\}$, while $c_{\infty}$ is the non-reactive fraction of total chlorine, i.e. the organic chloramines. Starting from the beginning of the pipe (the chlorination point) we have $x_{1}=0$, $t_{1}=t_{0}, \tau=t_{2}-t_{1}$ and we can drop the index from $x_{2}, t_{2}$. Giving

$$
c(x, t)=\left(c\left(0, t_{0}\right)-c_{\infty}\right) \cdot \mathrm{e}^{-K \tau}+c_{\infty} .
$$

\section{Experimental Work}

\section{1 "Torus" Pipe Rig}

All experiments discussed in this paper were performed on the "TORUS" pipe rig or with pipe rig water. The pipe rig consists of a $1.3 \mathrm{~km}, 110 \mathrm{~mm}$ inside diameter pipe distribution system built of approved construction material. It is mainly made of medium density polyethylene (MDPE) and is divided into three sections of approximate length $500 \mathrm{~m}, 400 \mathrm{~m}$ and $400 \mathrm{~m}$, respectively, which are 
buried 0.5-1.0 m underground. Measurements were taken at the inlet and at the three sample points along the rig.

The amperometric total chlorine meters use a range of 0 to $2 \mathrm{mg} / \mathrm{l}$ of free or total chlorine and have an accuracy of $\pm 0.05 \mathrm{mg} / \mathrm{l}$ at full scale deflection. They were recalibrated, both in zero position and span, at regular intervals.

Chlorine titrations allow a full speciation of chlorine. Replicate titrations were performed (i.e. 3 to 5 titrations of the same water sample) in order to minimise errors. After discarding outliers, the averages of the remaining titration values are used for the following considerations.

\subsection{Pipe Rig Experiments}

A series of step flow and step chlorine experiments was devised in order to obtain the relationship between chlorine concentration within the pipe, the initial conditions and flow rate. The experimental schedule is depicted in Fig. ??.

Apart from the step chlorine trials, the total chlorine residual at the pipe rig inlet was about $0.35-0.4 \mathrm{mg} / \mathrm{l}$. Step changes in flow were from $0.08 \mathrm{l} / \mathrm{s}$ to $0.3 \mathrm{l} / \mathrm{s}$, $0.45 \mathrm{l} / \mathrm{s}, 0.6 \mathrm{l} / \mathrm{s}$ or $0.9 \mathrm{l} / \mathrm{s}$. Typically, a step change in chlorine was from $0.4 \mathrm{mg} / \mathrm{l}$ to $0.65 \mathrm{mg} / \mathrm{l}$.

For bottle trials samples of the pipe rig inlet water were taken in bottles of volume $500 \mathrm{ml}$ or $1000 \mathrm{ml}$ and analysed by titrations. 


\section{Chlorine Meter Preprocessing}

Chlorine meters exhibit their own dynamics, and consequently preprocessing techniques are necessary to eliminate the unwanted signal.

Let us assume that the (averaged) titration values are normally more accurate than the chlorine meters and, further, that the error in the meter readings is due to a relatively slow meter drift. The aim of the correction procedure is to find this error signal by using titration results and then to adjust the meter reading values as appropriate. Since a titration is a bench analysis, titration values are unequally spaced and relatively sparse. However, if the error signal is only varying slowly, the Nyquist theorem is still satisfied (i.e. there are at least two samples per period of the error signal). Evidence for the validity of this assumption of slow variation is given in Maier (?).

The error function is calculated as the ratio between the titration data $c_{b}\left(x_{0}, t_{i}\right)$ and the chlorine meter data $c_{m}\left(x_{0}, t\right)$. Here $t_{i}$ denotes the unequally spaced time instants of the titrations; $t$ represents, for brevity, the equally and closely spaced time instants of the meter readings, and $x_{0}$ is the distance of the point of investigation from the inlet. Since we are, in this context, only concerned with data specific to each sampling point, the dependence on the distance is irrelevant and we will omit $x_{0}$ in this section. Thus, we obtain the error function $\epsilon_{M}\left(t_{i}\right)$ (subscript $M$ for multiplicative) as

$$
\epsilon_{M}\left(t_{i}\right)=\frac{c_{b}\left(t_{i}\right)}{\left.c_{m}(t)\right|_{t=t_{i}}}
$$


To reconstruct the error function between the time instants $t_{i}, \epsilon_{M}\left(t_{i}\right)$ is at first reverse-calibrated and then interpolated. Let $w$ recalibration windows $W_{j}$ be of the form

$$
W_{j}=\left[t_{j}^{r}+\delta, t_{j+1}^{r}-\delta\right]
$$

where $t_{j}^{r}$ is the $j$ th time of recalibration and $\delta$ is a small positive perturbation. Following eq. (7), the reverse-calibration factors are

$$
r\left(t_{i}\right)=\prod_{k=1}^{j} \frac{\left.c_{m}(t)\right|_{t=t_{k}^{r}-\delta}}{\left.c_{m}(t)\right|_{t=t_{k}^{r}+\delta}} \text { for } t_{i} \in W_{j} .
$$

Thus, the reverse-calibrated error signal $\epsilon_{M}^{R}\left(t_{i}\right)$ is

$$
\epsilon_{M}^{R}\left(t_{i}\right)=\epsilon_{M}\left(t_{i}\right) \cdot r\left(t_{i}\right)
$$

Finally, this error function is interpolated

$$
\epsilon_{M}^{I R}(t)=\sum_{i=1}^{m-1} \epsilon_{M}^{R}\left(t_{i}\right) \cdot \ell_{i}(t)
$$

where $\ell_{i}(t)$ denotes the linear interpolation function that is non-zero between $t_{i}$ and $t_{i+1}$ and $m$ is the number of titration times $t_{i}$ in the considered data. The superscript $I$ indicates that $\epsilon_{M}^{I R}(t)$ is an interpolated signal, the superscript $R$ refers to the reverse-calibration of eq. (9). The reverse-calibration is intended to inverse the effect of the recalibrations such that the chlorine reading directly after recalibration (at $t=t_{k}^{r}+\delta$ ) is the same as the reading just before recalibration 
(at $t=t_{k}^{r}-\delta$ ). Since the recalibrations guarantee that the meter is within the linear region of its scale the resulting error function $\epsilon_{M}^{R}\left(t_{i}\right)$ should adhere to the low frequency assumption made above.

Finally the error function $\epsilon_{M}^{I R}(t)$ is filtered, in accordance with the assumption of slow varying error, to remove any high frequency noise and smooth the signal. Thus, we obtain

$$
\epsilon_{M}^{I R F}(t)=\epsilon_{M}^{I R}(t) * b_{5}(t)
$$

where $*$ denotes the convolution sum of the discrete Fourier transform and $b_{5}(t)$ is the impulse response of a fifth order low pass (Butterworth) filter. The cut-off frequency of $0.3 \frac{1}{\text { day }}$ was chosen for a remaining absolute value of the Fourier transform of approximately 1-2 \% of its maximum value (cf. Fig. ??).

The corrected chlorine data $c_{c}(t)$ is then obtained by multiplication of the meter data $c_{m}(t)$ with the final error function $\epsilon_{M}^{I R F}(t)$

$$
c_{c}(t)=c_{m}(t) \cdot \epsilon_{M}^{I R F}(t)
$$

Typical examples of the correction obtained with this procedure are given in figs. ?? and ??. These figures show original meter data (dotted line), corrected data (dark line) and titration results (asterisks). In the total investigated time period the maximal absolute error is reduced from $0.28 \mathrm{mg} / \mathrm{l}$ to $0.13 \mathrm{mg} / \mathrm{l}$, the maximal mean square error from $0.075 \mathrm{mg} / \mathrm{l}$ to $0.029 \mathrm{mg} / \mathrm{l}$.

Other preprocessing methods were tested and are reported elsewhere (?). The method described in this paper has the best performance of those tested. 


\section{Determination of Decay Coefficients}

Two possible descriptions of monochloramine decay as in eq. (5) were studied.

1) $K=$ constant,

2) $K=k_{b}+\frac{k_{f} k_{w}}{R_{H}\left(k_{f}+k_{w}\right)}$, as used in EPANET.

To calibrate both models the unknown parameters $K$ and $c_{\infty}$ (case 1 ) or $k_{b}$,

$k_{w}$ and $c_{\infty}$ (case 2) have to be found such that the data fits a model according to eq. (5).

\subsection{Parameter Estimation for the Single Decay Coeffi- cient Model}

To find the single decay coefficient $K$ as given in case 1 , the solution of a nonlinear optimisation problem is necessary.

The problem is to find the minimum over $K$ and $c_{\infty}$ of

$$
J_{1}=\sqrt{\sum_{j=1}^{n_{1}} \sum_{i=1}^{n_{2}}\left(c_{c}\left(x_{j}, t_{i}\right)-g_{1}\left(j, i ; K, c_{\infty}\right)\right)^{2}},
$$

with

$$
g_{1}\left(j, i ; K, c_{\infty}\right)=\left(c_{c}\left(0, t_{0, i}\right)-c_{\infty}\right) \exp (-K \tau(i, j))+c_{\infty},
$$

where $c_{c}\left(x_{j}, t_{i}\right)$ is the corrected chlorine meter reading at time $t_{i}$ and distance $x_{j}$, and $\tau(i, j)$ is the travel time between $x_{j}$ and $x=0$ determined for the aliquot of water that is at time $t_{i}$ at $x_{j}$. The measurement $c_{c}\left(0, t_{0, i}\right)$ is the corrected meter reading at the inlet for the time when the aliquot of water that reaches $x_{j}$ at time 